\title{
An Overview of the various Smart Contract Platforms in Blockchain
}

\author{
Saveetha. $D^{1}$ and Maragatham. $G^{2}$ \\ ${ }^{1}$ SRM Institute of Science and Technology, SRM Na- \\ gar,Kattankulathur,Kancheepuram,Chennai,TN,India,603203 \\ saveethdesrmist.edu. in \\ ${ }^{2}$ SRM Institute of Science and Technology, SRM Na- \\ gar,Kattankulathur,Kancheepuram,Chennai,TN,India,603203 \\ maragatgesrmist.edu.in
}

\begin{abstract}
Blockchain is a simple and powerful platform for creating and deploying smart contracts .Its main aim is to remove the barriers in the deployment of blockchain especially for the financial sector. It provides the privacy and control required by them. It is called as smart contract because these are self-executable piece of codes which will execute when certain predefined conditions are satisfied without the need for a third party .These smart contracts are increasing in number day by day as more users are deploying them. In this paper we tried to give an overview of the various smart contract platforms, its features, its advantages and also its disadvantages.
\end{abstract}

Keywords:Blockchain, Smart Contract, Platforms.

\section{Introduction}

A blockchain is a distributed database secured by cryptography. It is immutable in nature. Due to its decentralized nature Blockchain Technology is emerging as an eminent technology. The main purpose for which blockchain was developed was to maintain transaction records without a Central Authority. Blockchain kept evolving with various versions of it like Blockchain 1.0, 2.0, 3.0. In Blockchain 2.0, Smart Contracts were introduced. Nick Szabo coined the term Smart contracts in 1994. According to Nick, "a smart contract is a computerized transaction protocol that executes the terms of a contract." Now-a-days with the advent of many new technologies, the purpose of smart contracts changed to be more like a general purpose computation [1]

\subsection{Smart Contracts}

Smart Contract is a small piece of computer program that is stored and automatically executed when predetermined terms and conditions are met in a blockchain. It is like a protocol. It can execute a contract without any help from third parties, just by checking whether the conditions stated in the contract have been met or not. The contract is visible to all, [9]because of the decentralized nature of blockchain and contains all the terms of a particular agreement, Once deployed it cannot be changed hence it is immutable. The blockchain will be updated once the execution of the smart 
contract is completed. Smart Contracts can be run on public and private blockchain except multichain. A Blockchain can be called as programmable blockchain if it supports the execution of smart contracts. Bitcoin is not a programmable blockchainbecause it does not support smart contracts[12][13]. Smart Contracts can be written on various platforms like Hyperledger Fabric, Corda and BigchainDB. Many languages like C++, Java Script, Viper, Serpent, Java, Solidity, Golang, Simplicity, and Rholang can be used to write smart contracts[11].

\subsection{Features of Smart Contract}

- Autonomy-It is not under control of anyone for the execution of the smart contracts

- Decentralized-Since there is no central authority it can be deployed on any system.

- Self-executing- when certain conditions are met in the smart contract the code gets executed automatically.

- Tamper proof-once coded it cannot be modified

- Transparency-the smart contract will be visible to all.

- Less cost-since it eliminates the intermediaries like banks, it is less expensive

- Eliminates intermediaries-no need of individuals to monitor the contract[10].

\subsection{Benefits of Smart Contract}

- Speed and accuracy: Smart contracts are processed very quickly without having to spend time processing paperwork and correcting the errors.

- It saves time: instead of taking days, a contract can be verified in minutes.

- Trust: Once the terms and conditions are met the smart contracts get executed and the records of those transactions are shared across participants.

- Security: Since the transaction records are connected to the previous and subsequent records on a distributed ledger, it is considered to be secure. Tampering is not possible because they need to change many records if one desires to do so.

- Remove Intermediaries: There are no intermediaries who check the agreement. It removes the manual work done.

- Reduced Cost: It saves money; there is no need to pay fees to any intermediaries (such as lawyers, real estate.

- Immutable: Once a smart contract has been deployed it cannot be modified.

\subsection{Applications of Smart Contract}

- Create own crytocurrency

- Create tokens

- Certificate verification

- Banking

- Financial Transactions

- Market prediction 
- IoT

- Domain name registration

- Escrow for purchase transaction

- Multi signature wallets

\section{2 .Smart Contract Platforms}

\section{ETHEREUM}

Itis a globally used smart contract platform. It can be used to develop any kind of decentralized application and also for providing Initial Coin Offerings [3].

\section{Characteristics:}

- Free

- Transactions are charged in gas.

- Solidity,Viper and Yul are the smart contract programming language.

\section{Drawbacks:}

- Network busy

- Expensive than other platforms

- Due to security many contracts exposed to hackers.

- Cannot patch security flaws

- Assets stolen is difficult to track and irrecoverable

\section{HYPERLEDGER}

The Hyperledger project was started by the Linux Foundation in 2015. It is an open-source platform. Its main aim was to support the development of block chain-based distributed ledgers. Hyperledger is co-developed by IBM,Intel and SAP Ariba. It is a private block chain that supports the execution of chain codes/smart contracts. A smart contract in fabric is called as chain code

Smart contracts are of two different types

- Installed smart contracts- before the network is launched, we will install business logic on the validators.

- On-chain smart contracts -we deploy the business logic as a transaction committed to the blockchain and then executed by the subsequent transactions [4].

Four Hyperledger frameworks support smart contracts. They are namely

- Hyperledger Iroha

- Hyperledger Sawtooth

- Hyperledger Burrow

- Hyperledger Fabric

Languages like Go, JavaScript (node.js), and Java can be sued to write chain codes. Two different types of chaincode are there.

- System chaincode

- Application chaincode 
System chaincode handles system-related transactions while Application chaincode manages application states of the ledger [4].

\section{Characteristics:}

- Free,Scalabe,Trust

- Permissioned membership

- Multilanguage support

- Good Performance

- Supports plug-in components

\section{Drawbacks:}

- No token system(i.e.)no coin or no cryptocurreny

- Each party needs a channel

- Privacy and versioning

\section{NEM}

Nem was launched in 2015. It is written in Java, so has few security vulnerabilities [5].

\section{Characteristics:}

- Language-Java

- Platform independent programming language

- Support many use cases than fintech

- Uses Proof of Importance(POI)as consensus algorithm

- NEM allows to make use of a private blockchain Mijin

- Excellent performance

- Can do 100 Transactions /sec

- Secure

- Highly scalable

\section{Drawbacks:}

- Development community is small

- Minimum tools

- It is less decentralized

- Lack of clarity on how to differentiate between Mijin fork and NEM blockchain.

- Lack of regular updates.

\section{WAVES}

Waves was introduced in June 2016 as a platform to facilitate token operations. It aimed to address the speed and scalability issues and it is open source [6].

\section{Characteristics:}

- Tokens,ICOs and crowdsales

- Written using Ride programming language

- No technical knowledge to create own tokens 
- Uses PoS

\section{Drawbacks:}

- Very few feature support

- Less user support

\section{STELLAR}

Stellar was introduced in 2014, so it is oldest platforms of all. It was developed by the Stellar Development Foundation. Stellar was used for international payments. IBM support[7].

\section{Characteristics:}

- $\quad$ Support ICOs

- High speed of transactions

- Less transaction cost than Ethereum

- Easy to use platform

- Support for multisignature

- Decentralized

- Simple and Easy to use

- Good performance

- Speed

- Security

\section{Drawbacks:}

- Difficult to use for complex smart contract.

- Minimum limit for having access.

- High competition

\section{NEO}

NEO can be used to execute smart contracts apart from being used for financial transactions as well. It can also be used as a platform for complicated transactions [8].

\section{Characteristics:}

- The consensus mechanism used is delegated Byzantine Fault Tolerance (dBFT).

- It uses two tokens namely NEO and GAS.

- The lightweight Virtual Machine, is known as NeoVM, T

- he virtual machine offered by NEO platform is efficient, certain \& scalable

- PKI support

- User-friendly platform with support of languages like java,python,C++

- 10,000 transactions per second

\section{Drawbacks:}

- Somewhat centralized

- Famous in China and Asia 
- High competition

- Lack of interoperability

- Regulatory uncertainty

\section{Conclusion}

Thus in this paper we have seen the details of the various smart contract platforms that are proficiently used nowadays highlighting the various pros/cons of each platform.

\section{References}

1. Gustavo, A.,Oliva Ahmed E.Hassan., Zhen Ming (Jack) Jiang.:An exploratory study of smart contracts in the Ethereum Blockchain platform. Empirical Software Engineering,vol.25,pp. 1864-1904(2020).

2. Nakamoto,S.:Bitcoin: A peer-to-peer electronic cash system.(2008)

3. Ethereum,https://ethereum.org,https://last accessed 2020/07/18.

4. Hyperledger,https://hyperledger.org, last accessed 2020/07/18.

5. Nem,https://nem.io/platforms, last accessed 2020/07/18.

6. .Waves,https://docs.waves.tech/en/building-apps/smart-contracts/waves-smart-contractsoverview\#orientation, last accessed 2020/07/18.

7. Stellar,https://www.stellar.org/developers/guides/walkthroughs/stellar-smartcontracts.html, last accessed 2020/07/18.

8. Neo,https://docs.neo.org/docs, last accessed 2020/07/18.

9. S. T. Ahmed and K. K. Patil, "An investigative study on motifs extracted features on real time big-data signals," 2016 International Conference on Emerging Technological Trends (ICETT), Kollam, 2016, pp. 1-4, doi: 10.1109/ICETT.2016.7873721.

10. K. D. Singh and S. T. Ahmed, "Systematic Linear Word String Recognition and Evaluation Technique," 2020 International Conference on Communication and Signal Processing (ICCSP), Chennai, India, 2020, pp. 0545-0548, doi: 10.1109/ICCSP48568.2020.9182044

11. Gunashree, M., Ahmed, S. T., Sindhuja, M., Bhumika, P., Anusha, B., \&Ishwarya, B. (2020). A New Approach of Multilevel Unsupervised Clustering for Detecting Replication Level in Large Image Set. Procedia Computer Science, 171, 1624-1633. https://doi.org/10.1016/j.procs.2020.04.174

12. K. Vijayakumar,C. Arun, "A Survey on Risk Assessment in Cloud Migration", International Journal of Applied Engineering Research, ISSN 0973-4562 Vol. 10 No.66 (2015)

13. M. Ananthi \& K. Vijayakumar, "Stock market analysis using candlestick regression and market trend prediction (CKRM)", Journal of Ambient intelligence and Humanised computing, April 2020. 
